\title{
Psychological effects of day case surgery compared with inpatient surgery
}

\author{
I R CAMPBELL, ${ }^{*} \mathrm{~J}$ M SCAIFE, $\dagger$ AND J M S JOHNSTONE*
}

${ }^{*}$ Leicester Royal Infirmary and †Towers Hospital, Leicester

SUMMARY Short term psychological disturbance in 70 children undergoing minor surgery was compared in two randomly allocated groups by means of questionnaires answered by their parents. Significantly less psychological disturbance was reported in children undergoing day case surgery compared with children admitted on the day before and discharged on the day after surgery. Children who had day case surgery were less often reported to require extra attention in the first week after discharge home, and at three months after operation these children were less often said to be still affected by their hospital stay. It is concluded that a reduced psychological upset can now be added to the other arguments in favour of day case surgery in children.

Treatment of children in hospital as day cases rather than inpatients has been advocated since the beginning of the century, ${ }^{1}$ and now up to $50 \%$ of general surgical operations in children are done in this way. ${ }^{2}$ Several arguments can be put forward in favour of this. Arguments of a clinical nature are earlier ambulation, leading to a possible decrease in morbidity, and the reduced risk of cross infection. Other arguments are lower cost of day case treatment, greater convenience for parents, and lastly but perhaps most importantly, reduced psychological upset of the child.

Fletcher has reviewed the whole question of psychological disturbance of children after admission to hospital. ${ }^{3}$ The existence of long term psychological upset after childhood admission to hospital was shown by Douglas in a large scale retrospective study of conduct disorder and emotional disorder in adolescents ${ }^{4}$; this was confirmed by Quinton and Rutter. ${ }^{5}$ In the shorter term, exact definition of psychological upset is difficult, but eating problems, sleeping problems, enuresis, regression to earlier levels of functioning, and anxieties about hospitals and illness are usually taken as indications of an adverse effect. Trials have been carried out to test various procedures in attempts to minimise psychological upset in children admitted to hospital; these trials have supported measures such as parents remaining with their children in hospital $^{67}$ and reduced length of hospital stay. ${ }^{8}$ On the whole, however, such trials have not been randomised. One exception is a trial by Von Troschke on children undergoing strabotomy, where a two day hospital stay with the mother staying in hospital was shown to produce less behavioural disturbance than a 10 day stay with only restricted visiting. ${ }^{8}$

We report a randomised trial in which children undergoing a general surgical operation were randomly allocated either to be admitted as day cases or to be admitted to hospital for two nights.

\section{Subjects and methods}

Subjects admitted to the study were children referred to the outpatient clinic, aged 9 months to 6 years, with no previous hospital admissions, and who required routine hospital admission for minor general surgical operations under general anaesthesia.

The children were randomly allocated to two groups - either to be admitted and discharged on the same day as the operation ('day cases') or to be admitted on the day before, and discharged on the day after the operation ('inpatients'). Randomisation was by drawing of a card from a prerandomised pile. Visits by a district nurse were arranged for all children to start on the day after discharge. Questionnaires were supplied to the parents and were to be completed on three occasions-before operation, one week after admission, and three months after admission. Each questionnaire contained about 100 questions covering the child's behaviour and response to the operation and several related matters. Some questions in the questionnaires were taken from standard questionnaires of child behaviour; the results of these questions are not 
reported here, but it is hoped to report them elsewhere. There are, however, no standard questionnaires specifically designed to determine adverse psychological effect on children from admission to hospital. Some questions were therefore devised especially for this study, and this was done on the basis of responses made in an open ended interview by a pilot sample of six parents not included in the subsequent study. For the purposes of the present report, responses to several important questions in the second and third questionnaires have been analysed. For each question in the questionnaires the parents were required to select from a small number of possible answers-for example, 'How much extra attention has s/he needed since the operation: a lot, some, or not very much?'

The main questionnaire (one week after operation) was sent to the parents of 70 children and was returned in 65 cases, (response rate 93\%). The questionnaire sent at three months after the operation was returned for 62 of these 70 children (response rate $89 \%$ ). Response rates for individual questions were in some cases slightly less. The children for whom data were not available did not differ in age, type of operation, or complication rate from the rest.

Seven children were inadvertently admitted or discharged on the wrong day (one of the day case group and six of the inpatient group). Data were available on all of these seven. Except for a preponderance of the inpatient group, these seven did not differ in any apparent way from the rest of the sample. Analysis of the data has been carried out both including and excluding these seven patients with no appreciable effect on any of the results of the study. It is therefore concluded that no error has been introduced by these breaches of protocol.

In a further seven cases it was necessary to override the protocol for medical reasons. In each case the child was kept in hospital for an extra 24 hours for a reason such as vomiting after the general anaesthetic or delay in micturition after a circumcision. Again, analysis of the data has been carried out both including and excluding these seven patients, and again none of the results is affected. The data presented in the results section are for those 58 children $(83 \%)$ for whom the protocol was accurately followed (except where medical reasons made this impossible) and for whom the questionnaire at one week after operation was returned.

Of these 58 patients, 35 were admitted as day cases and 23 were admitted as inpatients. The mean ages on admission for the day case and inpatient groups were 3 years 9 months and 3 years 4 months, respectively (overall range 9 months to 6 years 10 months). The two groups were also comparable for sex, operation, and complication rate. Roughly half of the children were circumcised, a quarter had a herniotomy performed, and the rest underwent a variety of procedures such as removal of a mass or cystoscopy. Four children developed complicationstwo inguinal wound infections and two scrotal haematomas - of which one was in the day case group and three were in the inpatient group (the difference was not significant).

The two groups were also comparable for the parents' assessments of amount of pain experienced after discharge, degree of difficulty in micturition, and amount of upset during dressing of any wound.

The significance of differences found was assessed using the $\chi^{2}$ statistic with Yates's correction where appropriate. ${ }^{9}$ Significance was taken to mean $\mathrm{p}<0.05$.

\section{Results}

Children treated as inpatients were found to be more affected by their hospital stay than children treated as day cases. At one week after discharge parents reported that eight of 23 inpatient children had 'needed a lot of extra attention since the operation' compared with one of 35 day case children $\left(\chi^{2}=10 \cdot 9, p<0 \cdot 01\right)$. The trend was still present at three months but was no longer significant $(p=0 \cdot 2)$. Furthermore, at three months five of the inpatient children were still affected by their hospital stay in their parents' opinion compared with none of the day case children $\left(\chi^{2}=5 \cdot 9\right.$, $\mathrm{p}<0 \cdot 025$ ).

As might be expected, the parents of children admitted as day cases were mostly able to stay with their children all the time that the children were on the ward (32 out of 35 ) compared with only a minority of the parents of the inpatient group (five out of 23$)\left(\chi^{2}=23 \cdot 6, p<0 \cdot 001\right)$.

Parents were asked at one week after discharge what length of stay they would have preferred for their children. One third (seven out of 23) of the parents of the inpatient children would have preferred a shorter stay and none a longer stay. Parents of four of the 35 day case children would have preferred a longer stay and three an even shorter stay $\left(\chi^{2}=6.9, p<0.05\right)$.

There were no extra demands for community care facilities in the day case group. This is being reported in detail elsewhere. No differences between the two groups were detected by the questionnaires in the remaining five points looked at-that is, in the degree of upset on admission to hospital, in the amount of postoperative pain, in the frequency 
of infection, or the difficulty with micturition after discharge home, and in the amount of upset during dressings of the wound.

\section{Discussion}

There have been many reports of psychological upset in children during and after admission to hospital; Fletcher has provided a comprehensive review. ${ }^{3}$ However, relatively few randomised controlled trials have been carried out. The disturbance to the child has been found in two (non-randomised) studies to be less if the parents sleep in the hospital with their child. ${ }^{6}{ }^{7}$ Many parents are unable to stay and sleep in the hospital, however, even if the opportunity is offered. The usual reason for this is responsibility for other children at home, but some parents are themselves afraid of the hospital environment and are reluctant to remain there any longer than is absolutely necessary.

A reduced length of hospital stay is another method of reducing separation of children from their parents. In the present study we attempted to assess any difference in upset between children admitted as day cases and children admitted for two nights by means of a randomised controlled trial using questionnaires. The questionnaires used appear to have been effective in detecting psychological effects in that the questions devised were able to detect significant differences between the two groups of children.

On the one hand, less upset might be expected in the shorter stay group because of a shorter exposure to the disturbing environment of the hospital; and on the other hand more disturbance might be expected in the shorter stay group if parental anxieties about care of the child discharged home soon after a general anaesthetic and surgical procedure were communicated to the child. In the event the shorter stay group was appreciably less affected by hospital admission. According to the parents' responses, inpatient children needed more attention on discharge home and were more likely to be still affected by their hospital stay three months later. The questions were designed to measure the child's behaviour or change in behaviour, and it is open to question whether the demonstration of a greater change in behaviour in the inpatient children is a valid method of showing a greater detrimental effect of hospital admission; however, this does seem the most compelling interpretation.
In view of the effect on the children staying in hospital for two nights, it is not surprising that one third of the parents of these children would have preferred a shorter stay in hospital. It is also worth noting that some of the parents of the children treated as day cases would also have preferred a shorter stay-the children were in hospital from 8 am to $5 \mathrm{pm}-$ but further shortening of the period of postoperative observation is controversial.

Apart from minimising the effect on the child, another reason for keeping hospital stay to a minimum is the reduction in cost that results, although precise figures are difficult to give. Other possible arguments for shorter hospital stays are reductions in lengths of waiting lists and reduction in cross infection.

On all these grounds it seems that efforts should be made to reduce hospital stay as far as possible. With regard to the psychological effect on the child, the point was brought home to us by several comments on the returned questionnaires of the longer stay children such as 'He's always saying "you left me on my own in hospital and went away.",

\section{References}

${ }^{1}$ Nicoll JH. The surgery of infancy. $B r$ Med $J$ 1909;ii:753-4.

2 Atwell JD. Changing patterns in paediatric surgery care. Ann $R$ Coll Surg Engl 1978;60:375-83.

${ }^{3}$ Fletcher B. Psychological upset in posthospitalized children: A review of the literature. Matern Child Nurs J 1981;10:185-95.

${ }^{4}$ Douglas JWB. Early hospital admissions and later disturbances of behaviour and learning. Dev Med Child Neurol 1975;17: 456-80.

${ }^{5}$ Quinton D, Rutter M. Early hospital admissions and later disturbances of behaviour: an attempted replication of Douglas' findings. Dev Med Child Neurol 1976;18:447-59.

${ }^{6}$ McGillicuddy MC. A study of the relationship between mothers rooming-in during their children's hospitalization and changes in selected areas of children's behaviour. Dissertation Abstracts International 1976;37:700B.

7 Couture CJ. The psychological response of young children to brief hospitalization and surgery: the role of parent-child contact and age. Dissertation Abstracts International 1976;37:1427B.

${ }^{8}$ Von Troschke J. Behaviour changes in children after a short period of hospitalisation: results of an empirical investigation. Zeitschrift für Psychotherapie und Medizinische Psychologie 1972;22:45-54.

9 Armitage P. Statistical methods in medical research. Oxford: Blackwell Scientific, 1971.

Correspondence to Dr IR Campbell, Mersey Regional Centre for Radiotherapy and Oncology, Clatterbridge Hospital, Bebington, Wirral, Merseyside L63 4JY.

Accepted 27 November 1987 